\title{
Analysis of Influence of UAS Speed Range and Turn Performance on Detect and Avoid Sensor Requirements
}

\author{
Devin P. Jack ${ }^{1}$, Jeremy Hardy ${ }^{2}$, and Keith D. Hoffler ${ }^{3}$ \\ Adaptive Aerospace Group, Hampton, Virginia, 23666
}

\begin{abstract}
In support of NASA's Unmanned Aircraft Systems Integration in the National Airspace System project and RTCA Special Committee 228, an analysis has been performed to provide insight in to the trade space between unmanned aircraft speed and turn capability and detect and avoid sensor range requirements. The work was done as an initial part of the effort to understand low size, weight, and power sensor requirements for aircraft that have a limited speed envelope or can limit the envelope for portions of their mission and may be able to turn at higher than "standard rate." Range and timeline reductions coming from limiting speed range and from increasing available turn rate in some speed ranges are shown.
\end{abstract}

\section{Nomenclature}

$\begin{array}{ll}\mathrm{CPA} & =\text { closest point of approach } \\ \mathrm{deg} & =\text { degrees } \\ d_{x} & =\text { range in the x-dimension } \\ d_{y} & =\text { range in the y-dimension } \\ \mathrm{dh} & =\text { vertical separation } \\ \mathrm{DMOD} & =\text { distance modifier } \\ \mathrm{ft} & =\text { feet } \\ \mathrm{h} & =\text { vertical separation threshold } \\ \mathrm{HMD} & =\text { horizontal miss distance } \\ \mathrm{KCAS} & =\text { knots calibrated airspeed } \\ \mathrm{KTAS} & =\text { knots true airspeed } \\ \mathrm{r} & =\text { horizontal range } \\ \dot{r} & =\text { horizontal closure rate } \\ \text { sec } & =\text { second } \\ t_{C P A} & =\text { time to closest point of approach }(\mathrm{CPA}) \\ t_{d h} & =\text { time to achieve vertical separation } \\ \tau_{m o d} & =\text { modified tau } \\ v_{r x} & =\text { relative velocity in the x-dimension } \\ v_{r y} & =\text { relative velocity in the y-dimension }\end{array}$

\section{Introduction}

The integration of unmanned aircraft systems (UAS) into the U.S.' National Airspace System (NAS) is highly desirable to many stakeholders across government, industry, and academia; maintaining the same level of safety currently in the NAS requires a means of replacing the manned aircraft pilot's obligation to see-and-avoid other aircraft. RTCA Special Committee 228 (SC-228), a federal advisory committee consisting of public and private stakeholders, is developing minimum technical requirements for a detect-and-avoid (DAA) system to be used in lieu of a manned aircraft pilot's see-and-avoid capability. A DAA system uses a suite of sensors, trackers, a detection algorithm, and display to provide the remotely located pilot with sufficient traffic situation awareness to avoid a loss

${ }^{1}$ Research and Development Engineer, 100 Exploration Way Ste 330, Hampton, Virginia, 23666, Member.

${ }^{2}$ Research and Development Engineer, 100 Exploration Way Ste 330, Hampton, Virginia, 23666, Member.

${ }^{3}$ President and Senior R\&D Engineer, 100 Exploration Way Ste 330, Hampton, Virginia, 23666, Associate Member. 
of separation with other aircraft. SC-228's recently published minimum operational performance standards (MOPS) for DAA systems are outlined in DO-365 [1], while the accompanying MOPS for air-to-air radar are outlined in DO366 [2]. To make the problem more manageable, DO-365 and DO-366 were necessarily developed to support a limited set of UAS performance and operations. Ongoing work is intended to enable more types of UAS and support more complete operations.

The development of DO-365 made assumptions on the speed range and maneuverability of the unmanned aircraft (UA). In current NAS operations, a turn rate of $3 \mathrm{deg} / \mathrm{sec}$ is considered a standard-rate turn and is expected by controllers for most maneuvers. For typical airspeeds in the NAS, this standard rate of turn keeps the accelerations experienced by the humans on-board to an acceptable level. To facilitate integration of UAS in the NAS, SC-228 asserted that UA must be capable of turning at a rate no less than $3 \mathrm{deg} / \mathrm{sec}$ and gave no credit to the UA for being able to turn faster. The assumed speed range limited UA operations to between 40 and 200 KTAS while below 10,000 $\mathrm{ft}$ above mean sea level (MSL). The worst case across the airspeed range drove the requirements giving no credit for a UA flying in a more limited speed range. Using these assumptions, range and time requirements were derived to define minimum sensor capability requirements and alert timing requirements.

With no humans on-board the UA, turn performance does not need to be constrained for pilot and passenger comfort. A UAS could reasonably operate using turn rates much greater than $3 \mathrm{deg} / \mathrm{sec}$, the standard rate for manned aircraft operations, but the current DO-365 requirements do not provide any benefit from turning faster. Similar to the approach used to derive the range and time requirements of the first edition of DO-365, a trade-space study has been performed to define the range and time requirements for a UA with improved turn rate capability. The results presented herein detail the range and time required to maintain DAA Well Clear as a function of sustained turn rate. The results are particularly applicable to sensors used to detect non-cooperative intruder aircraft. A non-cooperative aircraft is not equipped with an electronic means of broadcasting its state information either via an active surveillance transponder or an automatic dependent surveillance - broadcast out (ADS-B Out) system. Typically, non-cooperative sensors are much more limited in range capability than the cooperative sensors. The cost, size, weight, and power (C-SWAP) of non-cooperative sensors is very sensitive to the range at which established tracks are required; minimizing range will provide more flexibility in sensor development.

The range requirement presented in DO-365 and DO-366 are driven by the 200 KTAS upper-bound, while the time requirement is driven by the lower bound of 40 KTAS. The upper bound of 200 KTAS was set in order to ensure that air-to-air radar could detect non-cooperative aircraft with sufficient distance to remain safely separated. The lower end of the assumed speed range was defined to limit the need for excessively large alerting times; slow moving aircraft have difficulty achieving the needed separation. Currently, UAS must meet the requirements associated with these speed bounds, regardless of whether they plan to only operate in a reduced speed range. Introducing multiple 'bins' of operational speed ranges may enable more UA to safely integrate into NAS operations.

A key effort towards the development of DO-365 was the derivation of a quantitative definition of DAA Well Clear. U.S. Code of Federal Regulations Chapter 14, Part 91.113 (14 CFR 91.113) states that a pilot must remain well clear from other aircraft, but the definition of well clear is left to the pilot's judgment and experience [3]. DAA Well Clear is a separation standard developed for use by UAS to comply with 14 CFR and is expanded upon in the following section.

\section{A. DAA Well Clear}

Per 14 CFR 91.113 [3], "vigilance shall be maintained by each person operating an aircraft so as to see and avoid other aircraft. When a rule of this section gives another aircraft the right-of-way, the pilot shall give way to that aircraft and may not pass over, under, or ahead of it unless well clear.” Thus, utilizing an onboard pilot's ability to see and avoid traffic, an aircraft must remain 'well clear' from other vehicles at all times. With a pilot onboard, the definition of well clear is qualitative, based on the ability, judgment, and preferences of the pilot in command. Without an onboard pilot, a quantitative definition of well clear is required to establish separation requirements for unmanned aircraft DAA systems. A quantitative definition of well clear provides a repeatable target for which a DAA system may support safe separation from other aircraft by providing information to the pilot or to automation. The DAA Well Clear volume is intended to include interoperability principles with respect to Air Traffic Control (ATC) operations and current collision avoidance systems such as the Traffic alert and Collision Avoidance System (TCAS) [4]. To ensure interoperability with the current NAS, values used to define the DAA Well Clear volume must be large enough to avoid issuance of TCAS corrective resolution advisories [5] but not so large as to interfere with ATC separation services. This definition has been discussed and refined through a process involving NASA, Department of Defense (DoD), Federal Aviation Administration (FAA), and SC-228.

The proposed definition of a DAA Well Clear volume is represented using the following inequality [1]: 


$$
\left[0 \leq \tau_{\text {mod }} \leq \tau_{\text {mod }}^{*}\right] \text {.and. }\left[H M D \leq H M D^{*}\right] \text {. and. }\left[-h^{*} \leq d h \leq h^{*}\right]
$$

where HMD is the kinematic projection of the minimum horizontal miss distance in feet, and dh is the vertical separation between the two aircraft involved in the encounter. In Eqs. 1 through 4, the lack of a superscript denotes the instantaneous value at any given time during the encounter. The superscript * denotes the value given as a threshold in the quantitative definition of well clear. Table 1 shows the numeric values used to define the DAA Well Clear threshold.

The definition of modified tau, $\tau_{\text {mod }}$, is inherited from TCAS [5] and given in seconds by

Table 1. DAA Well-Clear definition.

\begin{tabular}{|l|c|l|l|}
\hline Parameter & Symbol & Units & Value \\
\hline Vertical Displacement & $\mathrm{h}^{*}$ & feet & 450 \\
\hline Modified Tau & $\tau_{\text {mod }}^{*}$ & seconds & 35 \\
\hline Horizontal Miss Distance & HMD* $^{*}$ & feet & 4000 \\
\hline
\end{tabular}

$$
\tau_{\text {mod }}=\frac{D M O D^{2}-r^{2}}{\dot{r} r}
$$

where TCAS defines DMOD as a horizontal distance threshold with varying values depending on the UA's altitude and $\mathrm{r}=$ horizontal range and $\dot{r}=$ horizontal range rate between the two aircraft. For the purposes of the DAA Well Clear definition, HMD* is used in place of DMOD in Equation 2.

The predicted horizontal miss distance is represented as:

$$
H M D=\sqrt{\left(d_{x}+v_{r x} t_{C P A}\right)^{2}+\left(d_{y}+v_{r y} t_{C P A}\right)^{2}}
$$

where $d_{x}$ is the horizontal separation in the x-dimension, $d_{y}$ is the horizontal separation in the y-dimension, $v_{r x}$ is the relative horizontal velocity in the $\mathrm{x}$-dimension, and $v_{r y}$ is the relative horizontal velocity in the $\mathrm{y}$-dimension. The parameter $t_{C P A}$ is the time to closest point of approach (CPA) and is calculated as:

$$
t_{C P A}=\max \left(0,-\frac{d_{x} v_{r x}+d_{y} v_{r y}}{v_{r x}^{2}+v_{r y}^{2}}\right)
$$

In Eq. 1, the horizontal separation requirements are segregated from the vertical separation requirements. In other words, if an aircraft is adequately separated in the vertical plane to avoid a loss of DAA Well Clear, the horizontal separation has no effect, and vice versa. Fig. 1 shows a simplified notional depiction of the DAA Well Clear definition, though its actual shape is more complex. The figure also separates horizontal (Fig. 1a) and vertical (Fig. 1b) separation requirements.

In the horizontal dimension, both time and distance constraints must be violated in order to have a loss of DAA Well Clear; whereas, in the vertical dimension, violation of only the distance constraint will result in a loss of DAA Well Clear (DWC).

In the horizontal dimension, as shown in Fig. 1a, the aircraft must remain outside of a time-based boundary $\left(\tau_{\text {mod }}^{*}\right)$ unless the projected minimum distance between the two aircraft is greater than the specified HMD*. The HMD is a kinematic projection using the velocity vectors of the ownship and intruder aircraft. Thus, the maneuvering aircraft must turn to a heading that achieves a projected value of HMD greater than HMD* to maintain DWC as opposed to being physically offset by a distance of HMD*. The angle that provides the required HMD projection varies greatly with the initial range between the encountering aircraft. 
In the vertical plane, as shown in Fig. 1b, the aircraft must remain outside of a spatial boundary defined by $\pm h^{*}$. In contrast to the horizontal separation requirements, the vertical separation is not a projection but an absolute vertical distance between the aircraft. Since the vertical boundary is fixed, the time required to reach the specified vertical separation $\mathrm{h}^{*}$, or $t_{d h}$, must be analyzed.

Note this paper focuses on the horizontal dimensions of DWC.

\section{B. Maneuver Reference Frame \\ When defining range and time requirements for} maneuvering to maintain DWC, it is important to consider the reference frame being used. The initial requirements presented in Appendix D of DO-365 [1] measure the range and time relative to the intruder aircraft, as shown in Fig. 2. This figure shows a notional depiction of the encounter geometry and DWC volume (dashed red). The figure indicates that the maneuver initiation range (MIR) and $t_{C P A}$ are measured relative to the intruder. Note that for simplicity the figure does not accurately depict the $t_{C P A}$ measurement, as this would more accurately be represented measured to the CPA point rather than the intruder position. This reference frame is of interest primarily for sensor measurements; an air-to-air radar directly measures the position of the intruder aircraft and has no means of detecting a DWC volume.

In contrast to the intruder-centric measurements shown in Fig. 2, the range and time may be measured relative to a loss of DAA well clear (LoWC) and written as $\mathrm{R}_{\mathrm{LowC}}$ and $\mathrm{t}_{\mathrm{LowC}}$ respectively. Fig. 3 shows a notional depiction of the range and time measured relative to the DWC volume. This reference frame is preferable for timing assessments primarily due to the fact that DO-365 alerting requirements are generally provided as X seconds prior to LoWC.

Of these four parameter definitions, the two of primary interest are the range measured with respect to the intruder aircraft, MIR, and the time measured relative to the DWC volume, $t_{\text {LoWC }}$. Using these two parameters, the sensor range requirements can be derived from the MIR, and the alerting timeline contribution of the UAS maneuver may be assessed using the $t_{\text {LoWC }}$. This reference frame is used herein and is proposed for subsequent versions of DO-365 and related documents.

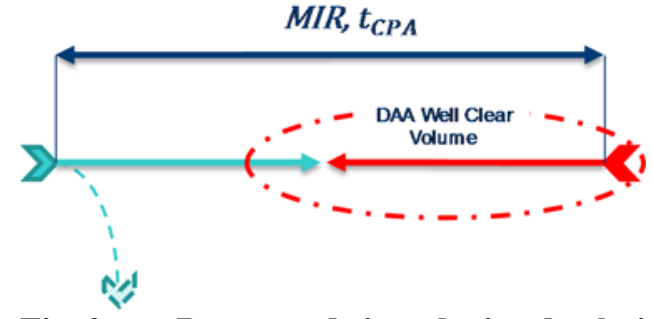

Fig. 2 Range and time depicted relative to intruder aircraft.

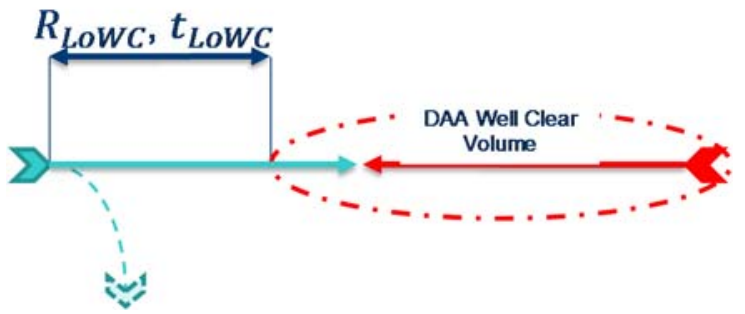

Fig. 3. Range and time depicted relative to DAA Well Clear Volume. 


\section{General Approach}

\section{A. Encounter Description}

The 2-degrees-of-freedom Prototyping Aircraft Interaction Research Simulation (2PAIRS) [6] tool was used to analyze pairwise encounters involving a maneuvering UA and a constant trajectory non-cooperative intruder. The two aircraft are initially flying on a direct collision course (CPA $=0$ feet). Initially trimmed to unaccelerated flight, the UA begins rolling into the specified turn rate immediately at the start of the simulation. The UA maintains the specified rate of turn until leveling out when a heading change of 90 degrees is achieved. The scenario continues until CPA has occurred and the aircraft are diverging.

A limiting factor in the design of a DAA system is the range capability of the air-to-air radar, the primary means of detecting a non-cooperative aircraft. The MIR is much less than the sensor capabilities for cooperative aircraft that actively broadcast their positions using ADS-B or an active surveillance transponder; therefore, cooperative intruders were not analyzed. For this reason, the intruder aircraft is modelled as a representative non-cooperative aircraft flying at $170 \mathrm{KTAS}$. This airspeed is found to be the 95th percentile airspeed for non-cooperative aircraft in the NAS [7]. Further, the encounters are setup to occur at 10,000 feet MSL, the maximum operating altitude for non-cooperative aircraft [3]. Using the maximum operating altitude provides the highest true airspeed when converting from calibrated airspeed (such as the statute speed limit).

\section{B. Experiment Design}

The UA turn rate and UA operational speed range are the primary independent variables of this study. For UA turn rate, integer values from standard rate ( $3 \mathrm{deg} / \mathrm{sec}$ ) up to $12 \mathrm{deg} / \mathrm{sec}$ were analyzed. The UA airspeed was varied from $20-250$ KCAS or about $25-291$ KTAS at 10,000 ft MSL. The lower bound of 20 KCAS is based on subject matter expertise and a survey of UA operator performance capabilities. The upper bound of 250 KCAS is the statute speed limitation for aircraft below 18,000 feet MSL [3].

An additional independent variable for this experiment is the initial time to CPA, $t_{C P A}$. Using the defined airspeeds and head-on geometry, time to CPA effectively defines the initial range between the two aircraft. This parameter was varied between 1 and 125 seconds in 1 second increments.

The primary metrics captured during each simulation run include a Boolean flag indicating a LoWC, the initial time to LoWC, and the initial positions of the UA and intruder aircraft. These four parameters were key to this analysis, but additional state information was captured using 2PAIRS.

The results presented herein are the minimum time and range the UA needs to maintain DWC and does not account for pilot response time, data link delay, control surface deflection delay, and other delays that may occur. Additional time and range must be added to account for these delays present during normal UA operations.

\section{Results}

The Maneuver Initiation Range, the range at which a maneuver must be initiated to avoid penetration of the DAA Well Clear boundary, as a function of UA turn rate and airspeed is presented in Fig. 4. Maneuver Initiation Range is shown in nautical miles on the y-axis, UA airspeed is shown in knots true airspeed (KTAS) on the x-axis, and UA turn rate is represented by the color of the line. The vertical dashed lines indicate the airspeed at which the UA is performing a maneuver at a bank angle greater than 60 degrees for the matching-color turn rate. A bank angle of 60 degrees is significant because it requires $2 \mathrm{~g}$ or more for level flight and the FAA classifies such maneuvers as aerobatic maneuvers [3]. Similarly, Fig. 5 shows the maneuver initiation time, the time at which a maneuver must be initiated to avoid a penetration of the DAA Well Clear boundary. 
The top curve (red) within Fig. 4 and Fig. 5 represents the time and range requirements for the initial edition of DO-365 but with the time reference standard proposed above. As can be seen in Fig. 4, the range requirement increases drastically as the airspeed decreases below 50 KTAS; analysis of this type of figure was used to limit the UA airspeed to between 40 and 200 KTAS in the initial edition of DO-365.

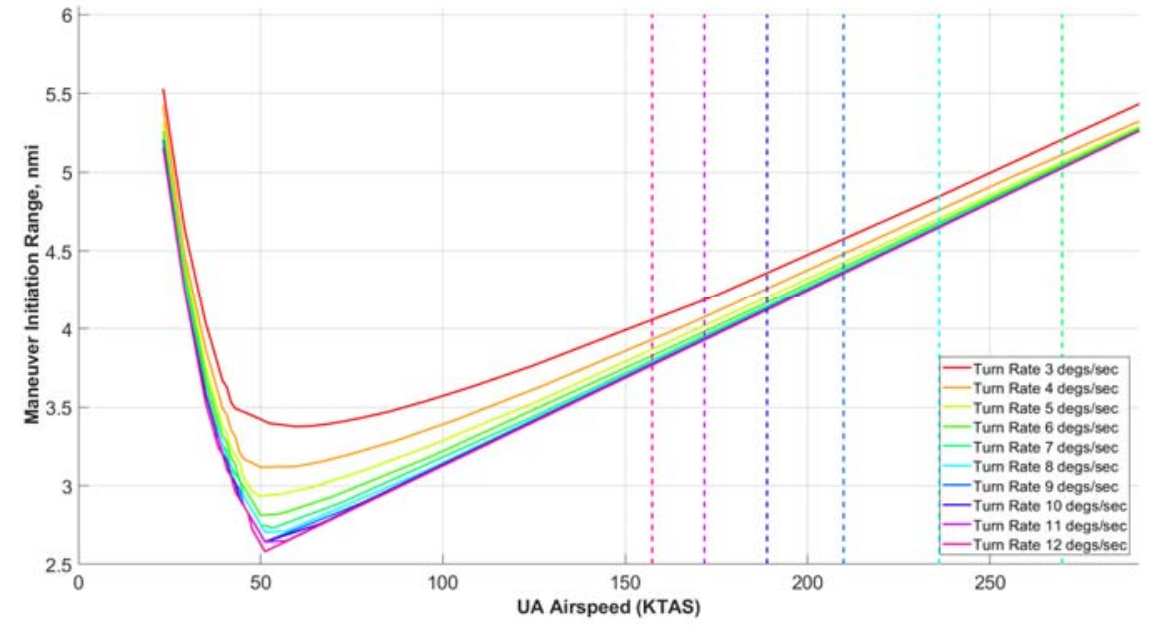

Fig. 4 Maneuver initiation range to remain DAA Well Clear vs. unmanned aircraft airspeed.

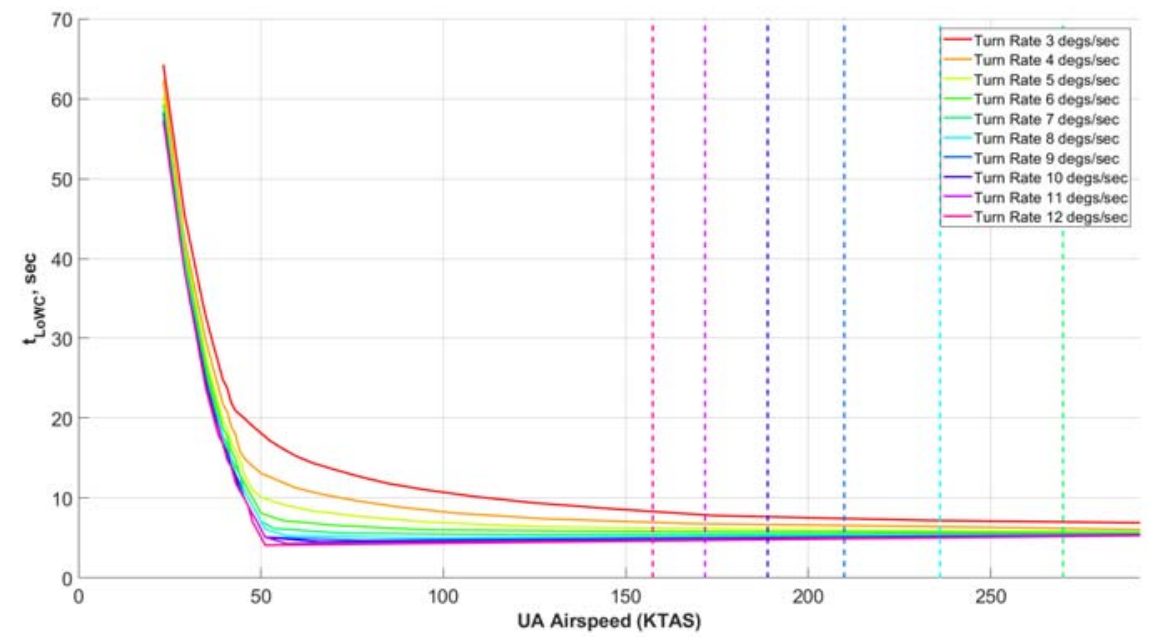

Fig. 5. Maneuver initiation time to LoWC to remain DAA Well Clear vs unmanned aircraft airspeed.

\section{A. Speed Range Effect}

One method to reduce the maneuver initiation range requirements below those in DO-365 and DO-366 is to further restrict the UA operational speed range. The assumed operational speed range of UAS in DO-365 is between 40 and 200 KTAS. The range requirement associated with the non-cooperative sensor is driven by the maximum MIR across that entire speed range, regardless of whether the applicant UA plans to operate across that entire range. An option to reduce the MIR requirement is to reduce the associated operational speed range. 
Table 2 shows the maneuver initiation range and time to LoWC required to maintain DWC for a variety of operational speed ranges. Table 2 captures the following speed ranges: less than 40 KTAS representing very slow moving aircraft whose operations focus on long endurance flights; 25-291 KTAS encompassing the statute speed range with a very low-end speed; 40-115 KTAS typical of a mid-size aircraft; 40-200 KTAS representing the current DO-365 operational speed range; and 45-75 KTAS representing an aircraft that intends to stay in a lower speed range.

Table 2 shows that the range and time required to maintain DWC increases for operational speed ranges that extended beyond the legacy DO-365 speed range of 40-200 KTAS. However, reducing the operational

Table 2 Effect of operational speed range on maneuver initiation range and time to LoWC.

\begin{tabular}{|c|c|c|}
\hline \multirow{2}{*}{$\begin{array}{c}\text { Operational } \\
\text { Speed Range } \\
\text { (KTAS) }\end{array}$} & \multicolumn{2}{|c|}{$3 \%$ sec } \\
\hline & MIR (nmi) & $t_{\text {LoWC }}(\mathrm{sec})$ \\
\hline $25-40$ & 5.26 & 59 \\
\hline $25-291$ & 5.43 & 59 \\
\hline $40-115$ & 3.68 & 24 \\
\hline $40-200$ & 4.47 & 24 \\
\hline $45-75$ & 3.47 & 20 \\
\hline
\end{tabular}
speed range within the legacy DO-365 bounds is an effective way of reducing range and time required to maintain DWC. The most restrictive speed range (45 to 75 KTAS) provides the most benefit in reducing the maneuver initiation requirements, but this speed range may be difficult to achieve during flight. Since $t_{L o W C}$ is monotonically decreasing as UA airspeed increases, the maximum $t_{\text {LoWC }}$ is driven by the low-end speed of any operational speed range. This same trend does not carry over to the MIR trend.

\section{B. Turn Rate Effect}

An alternative method of reducing the maneuver initiation range and time to LoWC is to credit aircraft capable of turning faster than $3 \mathrm{deg} / \mathrm{sec}$. In Fig. 54, the range required for aircraft at $100 \mathrm{KTAS}$ turning at $3 \mathrm{deg} / \mathrm{sec}$ is $3.57 \mathrm{nmi}$. If that aircraft operating at the same speed is capable of a $12 \mathrm{deg} / \mathrm{sec}$ turn (shown in Fig. 4 as a magenta line at the bottom), the range required for its non-cooperative sensor is reduced to $3.12 \mathrm{nmi}$. Similarly, in Fig. 5 the time to LoWC at which a maneuver must be initiated is reduced from $11 \mathrm{sec}$ to $4 \mathrm{sec}$. Table 3 shows the effect of increasing the turn rate assumption for three example aircraft speeds: 40, 100 and 200 KTAS. Within Table 3, the low speed aircraft require a significant amount of additional time to maintain DWC. The time associated with these low speed aircraft are driven primarily by the HMD* parameter of the DWC definition. Generally, these aircraft perform a complete 90 degree turn and continue flying perpendicular to the intruder aircraft in order to create the needed lateral separation of HMD* feet.

Table 3 Effect of turn rate on maneuver initiation range and time to LoWC.

\begin{tabular}{|c|c|c|c|c|c|c|}
\hline \multirow{2}{*}{$\begin{array}{c}\text { Turn Rate } \\
\text { (deg/sec) }\end{array}$} & \multicolumn{2}{|c|}{40 KTAS } & \multicolumn{2}{c|}{100 KTAS } & \multicolumn{2}{c|}{200 KTAS } \\
\cline { 2 - 7 }$(\mathrm{MIR}$ & $\boldsymbol{t}_{\text {LoWC }}$ & $\begin{array}{c}\text { MIR } \\
\text { (sec) }\end{array}$ & $\begin{array}{c}\boldsymbol{t}_{\text {LoWC }} \\
\text { (nmi) }\end{array}$ & $\begin{array}{c}\text { MIR } \\
\text { (nmi) }\end{array}$ & $\begin{array}{c}\boldsymbol{t}_{\text {LoWC }} \\
\text { (sec) }\end{array}$ \\
\hline 3 & 3.65 & 24 & 3.57 & 11 & 4.47 & 8 \\
\hline 7 & 3.24 & 17 & 3.18 & 5 & 4.26 & 5 \\
\hline 12 & 3.16 & 16 & 3.12 & 4 & 4.24 & 5 \\
\hline
\end{tabular}

\section{Effect of Turn Rate and Operational Speed Range}

The greatest reduction in MIR and $t_{\text {LoWC }}$ results from combining a more restrictive operational speed range and increased turn rate assumptions. Table 4, shown below, shows the MIR and $t_{\text {LowC }}$ required to maintain DWC for several combinations of turn rate and operational speed range. In general, range and time requirements are inversely proportional to turn rate.

As previously discussed and shown here to be true for all of the presented turn rates, the maximum $t_{\text {LoWC }}$ is driven by the low-end speed of any operational speed range. 


\section{Application}

As previously discussed, increasing the turn rate assumption and modifying the operational speed range are both

Table 4 Effect of operational speed range on maneuver initiation range and time to LoWC for 3,7 , and $12 \mathrm{deg} / \mathrm{sec}$ turn rates.

\begin{tabular}{|c|c|c|c|c|c|c|}
\hline \multirow{2}{*}{$\begin{array}{c}\text { Operational } \\
\text { Speed Range } \\
\text { (KTAS) }\end{array}$} & \multicolumn{2}{|c|}{$\mathbf{3}^{\circ} /$ sec } & \multicolumn{2}{c|}{$\mathbf{7}^{\circ} /$ sec } & \multicolumn{2}{c|}{$\mathbf{1 2}^{\circ} /$ sec } \\
\cline { 2 - 7 }$(\mathbf{M I R}$ & $\begin{array}{c}\boldsymbol{t}_{\text {LoWC }} \\
\text { (sec) }\end{array}$ & $\begin{array}{c}\text { MIR } \\
\text { (nmi) }\end{array}$ & $\begin{array}{c}\boldsymbol{t}_{\text {LoWC }} \\
\text { (sec) }\end{array}$ & $\begin{array}{c}\text { MIR } \\
\text { (nmi) }\end{array}$ & $\begin{array}{c}\boldsymbol{t}_{\text {LoWC }} \\
\text { (sec) }\end{array}$ \\
\hline $25-40$ & 5.26 & 59 & 4.94 & 53 & 4.88 & 52 \\
\hline $25-291$ & 5.43 & 59 & 5.27 & 53 & 5.26 & 52 \\
\hline $40-115$ & 3.68 & 24 & 3.33 & 17 & 3.29 & 16 \\
\hline $40-200$ & 4.47 & 24 & 4.26 & 17 & 4.24 & 16 \\
\hline $45-75$ & 3.47 & 20 & 2.96 & 12 & 2.88 & 10 \\
\hline
\end{tabular}

effective methods of reducing the maneuver initiation range and time to LoWC. An initial attempt to define UAS performance groups based on this analysis is presented in Fig. 6. The figure shows the MIR from Fig. 4 overlaid with four UAS performance groups: low-speed group, a mid-speed group, the legacy DO-365 operational speed range, and a high-speed group.

On the low end of the operational speed range, Fig. 6 depicts a low-speed group operating below 40 KTAS. Aircraft operating within this speed range are generally characterized as high-altitude, long-endurance aircraft with very sleek profiles when not maneuvering. Since these aircraft take such a significant amount of time and distance in order to maintain DWC, special considerations with respect to the alerting timeline and required separation are needed.

As seen in Table 4, certain speed ranges have a greater benefit to increasing the turn rate beyond the standard 3 deg/sec; specifically, the mid-speed ranges of 40-115 KTAS and 45-75 KTAS. Fig. 6 shows the mid-speed group encompassing 40-115 KTAS. Increasing the minimum turn rate from 3 to $7 \mathrm{deg} / \mathrm{sec}$ may provide sufficient reduction in non-cooperative sensor requirement so as to enable an increased number of aircraft to operate within this speed range. There is limited benefit of increasing the turn rate beyond $7 \mathrm{deg} / \mathrm{sec}$. Increasing the turn rate assumption to 12 $\mathrm{deg} / \mathrm{sec}$ may be more difficult to achieve in terms of system design of the UAS with limited additional benefit 
For aircraft capable of carrying non-cooperative sensors that outperform the minimum requirements specified in DO-366, there may be added operational benefit to flying at speeds greater than 200 KTAS. This high-speed group requires greater MIR than the legacy DO-365 performance group. This group differs from the other UAS performance groups since these UAS can be characterized as having greater system performance than those in the legacy DO-365 system; whereas the low- and mid-speed performance groups are unable to meet legacy DO-365 performance requirements. The recommended performance groups are designed to enable a higher number and variety of UAS to successfully integrate into current NAS operations.

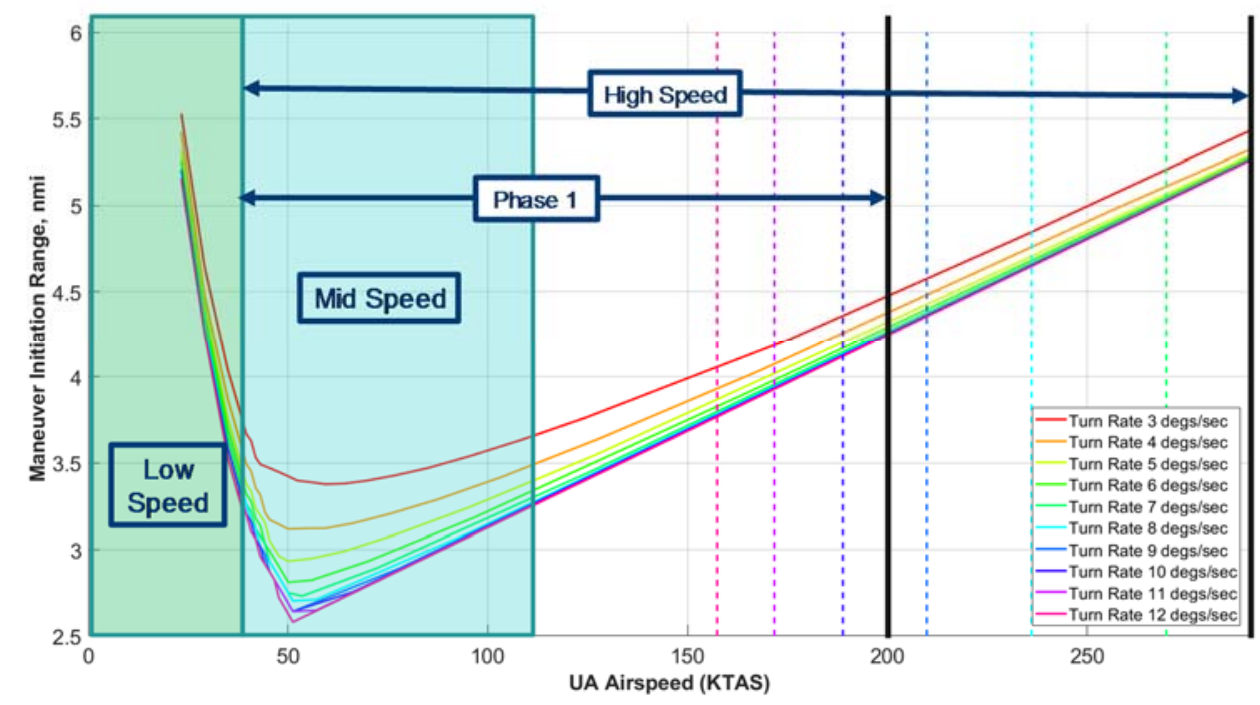

Fig. 6 Recommended UAS performance groups.

\section{Conclusion}

To enable the safe integration of an increased number and variety of UAS in to the U.S.' NAS, this paper outlines two methods of reducing the minimum range and time at which a maneuver must be initiated to maintain a UASspecific separation standard, termed DAA Well Clear. The two methods for reducing these requirements outlined were increasing the assumed minimum turn rate for UAS and modifying the operational speed range. Beyond showing the impact of these two methodologies, a series of recommend groups based on UAS operational speed range and turn rate were outlined. 


\section{References}

[1] RTCA Special Committee 228, "DO-365: Minimum Operational Performance Standards (MOPS) for Detect and Avoid (DAA) Systems,” Standards DO-365, RTCA Inc., Washington, D.C., 2017.

[2] RTCA Special Committee 228, "DO-366: Minimum Operational Performance Standards (MOPS) for Air-to-Air RADAR for Traffic Surveillance,” Standards DO-366, RTCA Inc., Washington, D.C., 2017.

[3] Federal Aviation Administration, “14 CFR 91 General Operating and Flight Rules,” Federal regulations, Department of Transportation, Washington, D.C., Apr. 2018. URL https://www.ecfr.gov/cgibin/retrieveECFR?gp=\&SID=de5f9357d1e2716832f6ca8b6f84244a\&mc=true\&n=pt14.2.91\&r=PART\&ty=HTML

[4] Consiglio, M., Chamberlain, J., Munoz, C., and Hoffler, K., 2013, 'Concept of Integration for UAS Operations in the NAS,' ICAS 2012-28th Congress of the International Council of the Aeronautical Sciences; 23-28 Sept. 2012; Brisbane, Australia.

[5] Federal Aviation Administration, “Introduction to TCAS II," version 7, November 2000.

[6] Jack, D. P., Hoffler, K. D., and Johnson, S.C., "Exploration of the Trade Space Between UAS Maneuver Performance and SAA System Performance Requirements,” NASA CR-2014-218264, May 2014.

[7] Kochenderfer, M., et. al., “Uncorrelated encounter model of the National Airspace System version 1.0,” MIT Lincoln Laboratory, Project Report ATC-345, 2008. 